\title{
IL-33 regulates the IgA-microbiota axis to restrain IL-1 $\alpha$-dependent colitis and tumorigenesis
}

\author{
Ankit Malik, ${ }^{1}$ Deepika Sharma, ${ }^{1}$ Qifan Zhu, ${ }^{1,2}$ Rajendra Karki, ${ }^{1}$ Clifford S. Guy, ${ }^{1}$ Peter Vogel, ${ }^{3}$ and Thirumala-Devi Kanneganti ${ }^{1}$ \\ 'Department of Immunology, St. Jude Children's Research Hospital, Memphis, Tennessee, USA. Integrated Biomedical Sciences Program, University of Tennessee Health Science Center, Memphis, Tennessee, \\ USA. ${ }^{3}$ Animal Resources Center and the Veterinary Pathology Core, St. Jude Children's Research Hospital, Memphis, Tennessee, USA.
}

\begin{abstract}
Inflammatory bowel diseases (IBD) affect over 5 million individuals in the industrialized world, with an increasing incidence rate worldwide. IBD also predisposes affected individuals to development of colorectal cancer, which is a leading cause of cancer-related deaths in adults. Mutations in genes encoding molecules in the IL-33 signaling pathway are associated with colitis and colitis-associated cancer (CAC), but how IL-33 modulates gut homeostasis is unclear. Here, we have shown that II33-deficient mice are highly susceptible to colitis and CAC. Mechanistically, we observed that IL-33 promoted IgA production from B cells, which is important for maintaining microbial homeostasis in the intestine. II33-deficient mice developed a dysbiotic microbiota that was characterized by increased levels of mucolytic and colitogenic bacteria. In response to chemically induced colitis, this microbial landscape promoted the release of IL-1 $\alpha$, which acted as a critical driver of colitis and CAC. Consequently, reconstitution of symbiotic microbiota or IL-1 $\alpha$ ablation markedly ameliorated colitis susceptibility in //33deficient animals. Our results demonstrate that IL-33 promotes IgA production to maintain gut microbial homoeostasis and restrain IL-1 $\alpha$-dependent colitis and CAC. This study therefore highlights modulation of IL-33, IgA, IL-1 $\alpha$, and the microbiota as a potential therapeutic approach in the treatment of IBD and CAC.
\end{abstract}

\section{Introduction}

Inflammatory bowel diseases (IBD), such as Crohn's disease (CD) and ulcerative colitis (UC), affect approximately 1.6 million individuals in the USA and 3.5 million individuals in Europe, constituting a major health problem $(1,2)$. IBD involves chronic inflammation in the intestine with episodes of acute flare-ups that manifest as abdominal pain, diarrhea, rectal bleeding, and body weight loss $(1,2)$. The mucus and the epithelial layer form a physical barrier between the microbiota and underlying host immune system, and deterioration of this barrier is prominent in IBD patients $(3,4)$. In addition, rodent studies have linked disruption of the epithelial barrier to cytokine imbalances in the gut that eventually lead to the development of IBD (5). IBD also predispose patients to the development of colorectal cancer, referred to as colitis-associated cancer (CAC) (6). Colorectal cancer is a leading cause of adult cancer-related deaths, with 160,000 cases being diagnosed annually in the USA, and colitis is associated with approximately $90 \%$ of these cases (7).

IL-33 is a member of the IL-1 cytokine family that is expressed constitutively in epithelial cells and reticular fibroblasts and can be induced in immune cells (8). IL-33 is usually localized in the nucleus and released upon cell death (9). However, unlike IL-1 $\beta$ and IL-18, which require cleavage by caspase 1 for bioactivity, IL-33 is bioactive in the full-length form (9, 10). IL-33 serves as an amplifier of the type 2 cytokine response from $\mathrm{T}_{\mathrm{H}} 2$, ILC2, mast cells, and alternatively activated macrophages during asthma, allergic hypersensitivity, and helminth

Conflict of interest: The authors have declared that no conflict of interest exists. Submitted: May 18, 2016; Accepted: September 15, 2016.

Reference information: / Clin Invest. 2016;126(12):4469-4481. doi:10.1172/JCI88625. infection (11-15). In addition, IL-33 has been reported to promote antiviral responses by enhancing cytokine production from cytotoxic T cells (16) and to exacerbate arthritis by hyperactivating mast cells (17).

Multiple studies have demonstrated that IL-33, ST2 (the binding site for IL-33), and soluble ST2 (sST2, a decoy receptor) are upregulated in the mucosa of patients with IBD, and polymorphisms in IL33 and ST2 have been associated with the development of IBD (18-22). Expression levels of ST2 correlate inversely with the grade of colon tumors, suggesting that IL-33 signaling is protective in CAC (22). However, the role of IL-33 in IBD and colon tumorigenesis is unclear. Genetic ablation of $I l 33$ in mice of a mixed genetic background lowered clinical symptoms in the early phase of experimental colitis, but also delayed the resolution of inflammation (23). Administration of recombinant IL-33 was also shown to ameliorate colitis in Il33-sufficient (WT) mice, suggesting a protective role for IL-33 in colitis $(24,25)$. By utilizing $\mathrm{I} l 33^{-/}$mice generated on a congenic C57BL/6 background and employing cohousing strategies and littermate controls, we show that IL-33 protected from colitis and CAC. IL-33 was critical to maintaining gut homeostasis by regulating IgA production in the colon. Reduced intestinal IgA in $\mathrm{Il33}^{-/-}$mice led to a dysbiotic microbiota characterized by increased levels of mucolytic and colitogenic bacteria. Consequently, administration of dextran sulfate sodium (DSS) in $\mathrm{Il33}^{-/}$mice led to increased release of proinflammatory IL-1 $\alpha$, which was a critical driver of CAC. Remarkably, cohousing Il33-/- mice with WT controls equilibrated the level of mucolytic bacteria and thereby protected $l l 33^{-}$ mice from colitis and epithelial hyperplasia. These findings reveal a critical role for IL-33 in regulating the immune responses and microbiota in the gut. 
A
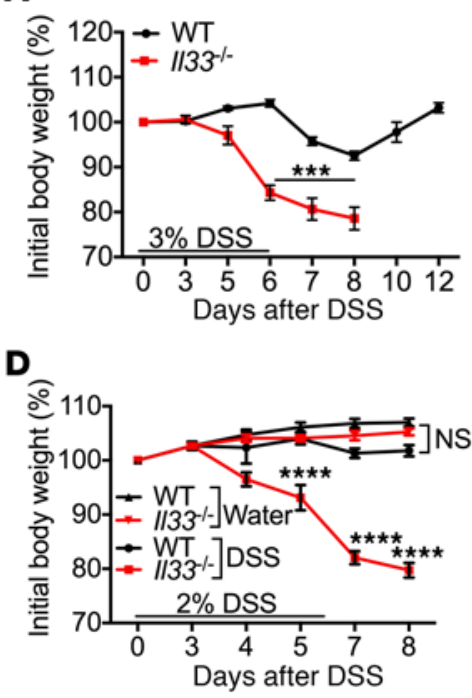

B

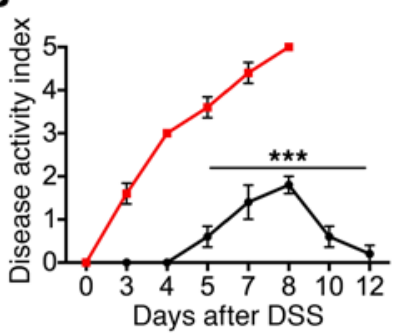

E

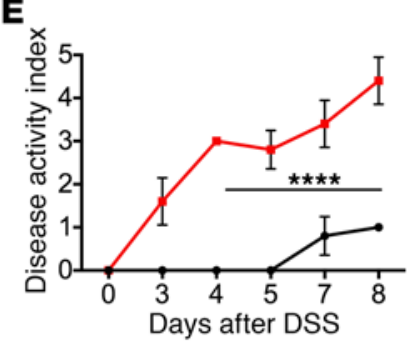

C

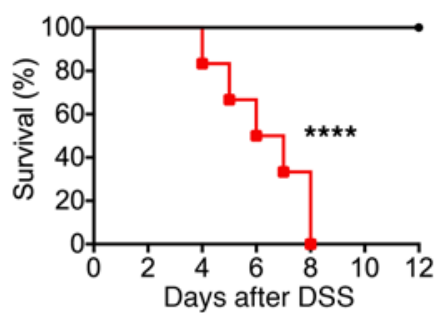

$\mathbf{F}$

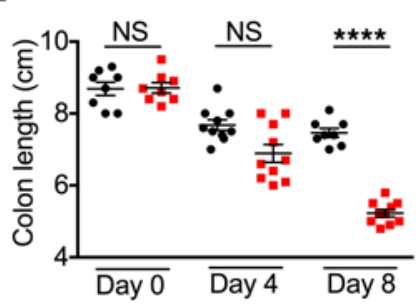

G

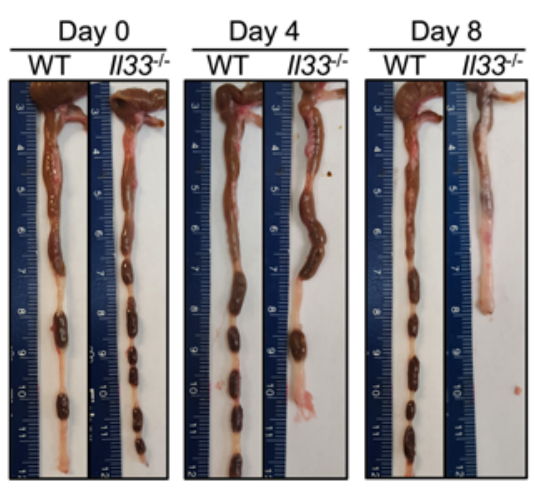

I

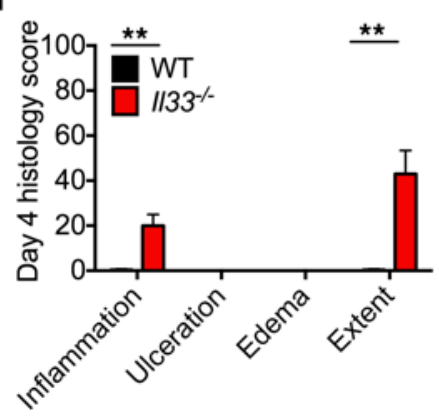

H

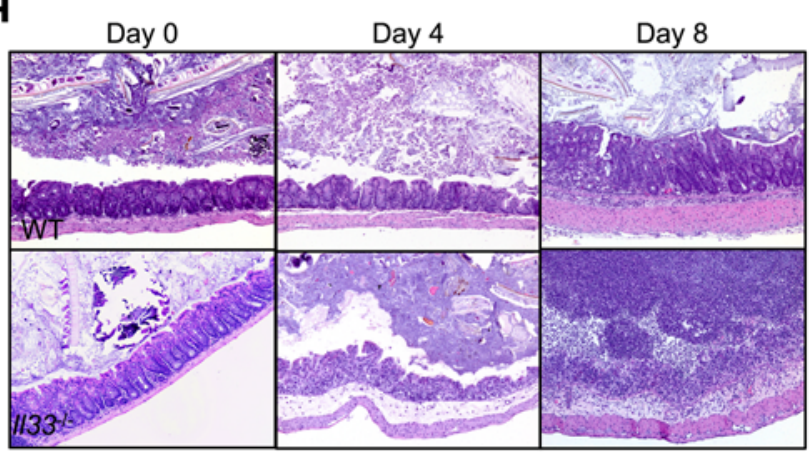

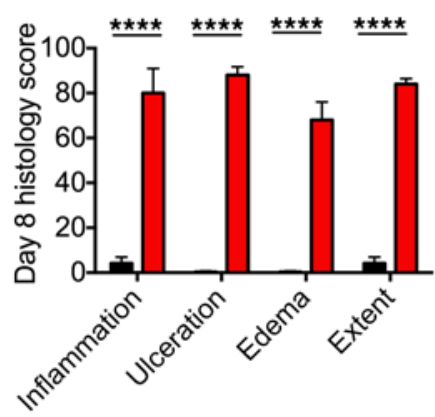

J



Figure 1. IL-33 decreases susceptibility to DSS-induced colitis. (A) Body weight change, (B) disease activity index, and (C) relative survival of WT and $1 / 33^{-1-}$ mice during administration of 3\% DSS in drinking water. (D) Body weight change and (E) disease activity index of mice during administration of $2 \%$ DSS in drinking water. (F) Colon length measurement and (G) representative pictures of colons from WT and $1 / 33^{-1-}$ mice at the indicated days after $2 \%$ DSS administration. (H) H\&E staining. Original magnification, $\times 10$. (I) Histological analysis of colon tissue from mice at the indicated days after $2 \%$ DSS administration. (J) Incidence and levels of bacteria in mesenteric lymph nodes (MLN) of mice at day 4 and day 8 after $2 \%$ DSS administration. Data represent 2 independent experiments and are presented as mean \pm SEM (A-I) or median (J). Each symbol represents an individual mouse. $n=8-10$ mice per time point per group. Data were analyzed by 2-way ANOVA (A, B, D, and E), log-rank (Mantel-Cox) test (C), or Kruskal-Wallis test (F and J) followed by Holm-Šídák post test. ${ }^{* *} P<0.01 ;{ }^{* *} P<0.001 ;{ }^{* * *} P<0.0001$.

\section{Results}

IL-33 regulates the development of colitis and associated cancer. To determine the role of IL-33 in pathogenesis of IBD, WT and Il33/- mice were administered with DSS in drinking water for 6 days, followed by regular drinking water. Following administration of $3 \%$ DSS, $I l 33^{-/-}$animals displayed significantly greater body weight loss and disease activity score when compared with WT mice (Figure 1, A and B). Further, $100 \%$ of the Il33/mice reached humane end point of the experiment $(>20 \%$ body weight loss) by day 8 , while all the WT mice survived (Figure 1C). At a lower dose of DSS (2\%), Il33-/- mice still displayed significantly greater weight loss and disease activity scores (Figure 

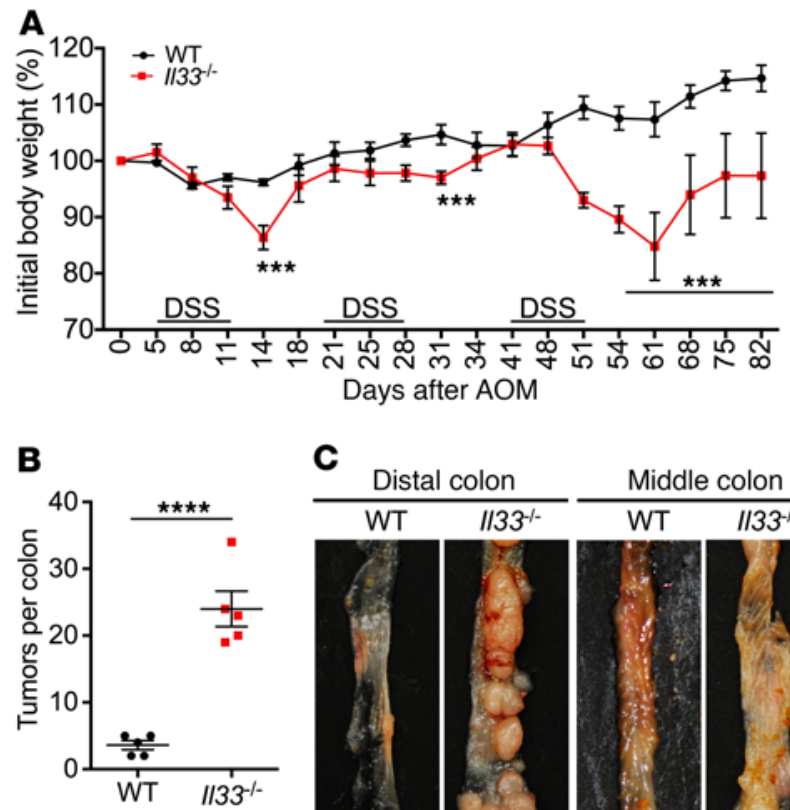

C Distal colon
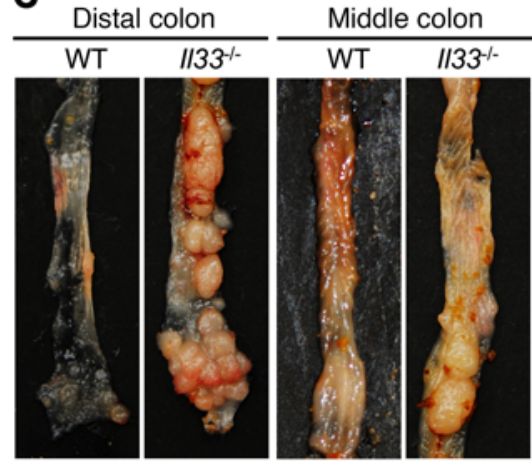

D
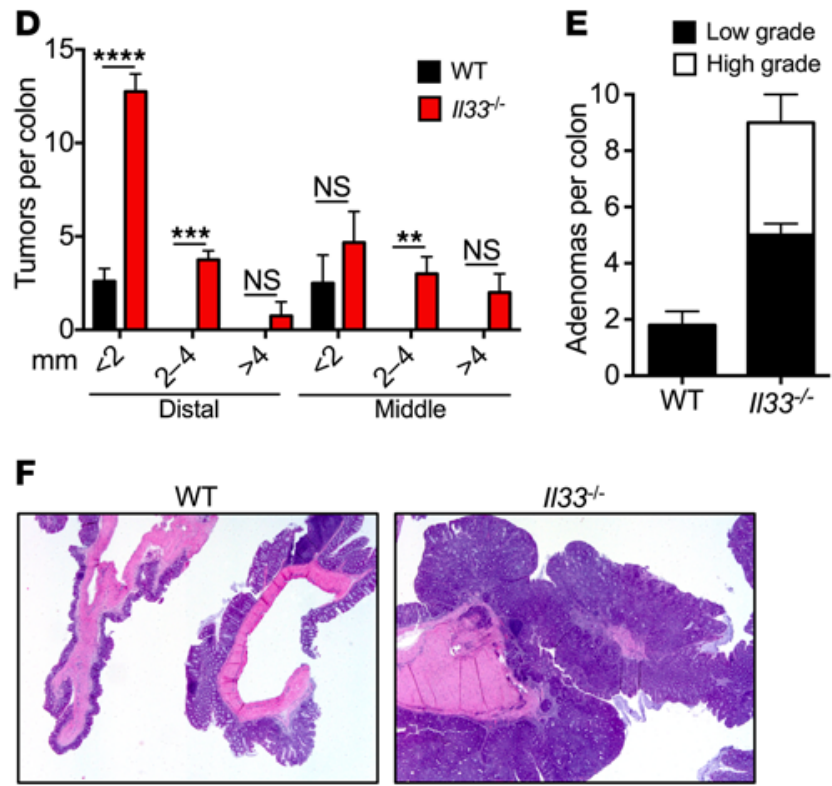

$1, \mathrm{D}$ and $\mathrm{E})$. Shortening of colon length is a feature of acute colitis (26), and $\mathrm{Il33^{-/- }}$ mice had significantly shorter colons at day 8 (Figure 1, F and G). Histological analysis revealed progressive increase in inflammation, ulceration, edema, and hyperplastic proliferation of epithelial cells in the colons of $I l 33^{-/-}$mice during DSS administration (Figure 1, H and I). Breakdown of the epithelial barrier in $\mathrm{Il33^{-/- }}$ mice was associated with dissemination of bacteria to the draining lymph nodes by day 8 (Figure 1J). Together, these data demonstrate that IL-33 ameliorates susceptibility to DSS-induced colitis.

IBD is a strong risk factor for development of CAC (6). To determine the role of IL-33 in development of chronic colitis and CAC, we utilized an established model of CAC wherein
Figure 2. IL-33 decreases susceptibility to CAC. (A) Body weight loss of WT and $1 / 33^{-1-}$ mice injected with AOM on day 0 and administered 3 rounds of $2 \%$ DSS in drinking water. (B and $\mathbf{D})$ Quantification of the number of tumors in the colon at day 82 after AOM injection. (C) Representative images of distal and middle colon at day 82 after AOM administration. (E) Histological analysis and (F) H\&E staining of colon sections at day 82 after AOM injection. Original magnification, $\times 2$. Data represent 2 independent experiments and were analyzed by 2-way ANOVA followed by Holm-Šídák post test (A) or Mann-Whitney $U$ test (B). Error bars represent mean \pm SEM. (A, B, and $\mathbf{D}$ ) and each symbol represents an individual mouse with 5 mice per group. ${ }^{* *} P<0.01 ;{ }^{* *} P<0.001 ;{ }^{* * *} P<0.0001$.

mice were injected with DNA damaging agent azoxymethane (AOM), followed by 3 rounds of low-dose (2\%) DSS treatment (27). Il33-/- mice lost significantly more body weight during each round of DSS treatment (Figure 2A). At the end of the experiment, WT mice exhibited few small tumors restricted to the distal colon (Figure 2, B-D). In contrast, Il33-/- mice exhibited a significantly increased number of tumors that were of a larger size and distributed throughout the distal and middle colon (Figure 2, B-D). Histological analysis revealed few low-grade adenomas in the colons of WT mice, whereas both low- and high-grade adenomas were observed more frequently in the colons of $I l 33^{-/-}$mice (Figure 2, E and F). These data demonstrate that IL-33 protects mice from colitis and CAC.

Lack of IL-33 leads to increased secretion of IL-1 $\alpha$ during DSS administration. To determine the immunological basis of increased colitis and CAC in $\mathrm{Il33}^{-/-}$mice, cytokine and chemokine production were analyzed in the colon during DSS administration. Day 4 was considered as a preclinical time point because there was no difference in body weight loss between WT and $\mathrm{Il33}^{-1-}$ mice at that time, while day 8 was considered an acute time point because significant differences in weight change and disease activity score were observed (Figure 1D). In the colon explants, significantly increased levels of IL-1 $\alpha$ were observed at both the preclinical time point (day 4) and acute time point (day 8) during DSS administration (Figure 3A). In contrast with the early increase in IL-1 $\alpha$, there was no difference in levels of the neutrophil chemokine $\mathrm{KC}$ (also known as CXCL1), granulocyte-CSF (G-CSF), IL-6, IL-1 $\beta$, macrophage inflammatory protein- $1 \alpha$ (MIP- $1 \alpha$, also known as CCL3), IL-10, and IFN- $\gamma$-induced protein 10 (IP-10, also known as CXCL10) at day 4 , while they were significantly enhanced at day 8 (Figure 3A and Supplemental Figure 1; supplemental material available online with this article; doi:10.1172/JCI88625DS1). Levels of other cytokines, such as IL-17, granulocyte-macrophage CSF (GM-CSF), monocyte chemoattractant protein-1 (MCP-1), TNF, and CCL5, were unchanged during the course of the experiment (Supplemental Figure 1). These data suggest that the release of IL-1 $\alpha$ precedes the enhanced inflammatory response in $I l 33^{-/-}$mice in response to DSS administration. While IL-1 $\alpha$ was upregulated at protein and transcript levels by day 8 , the levels of IL-1 $\alpha$ protein and transcript were similar between WT and $\mathrm{Il33^{-/- }}$ mice at day 4 during DSS administration (Figure 3, B and C). Therefore, DSS administration leads to the release of the preformed pool of IL- $1 \alpha$ early during disease progression.

To determine the cellular source of IL-33 and IL-1 $\alpha$ in the colon, we assessed for expression of Il33 and Il1a in epithelial $\left(\right.$ Epcam $\left.^{+} \mathrm{CD} 45^{-}\right)$and immune cells $\left(\mathrm{CD} 45^{+}\right.$Epcam $\left.^{-}\right)$from the 
A
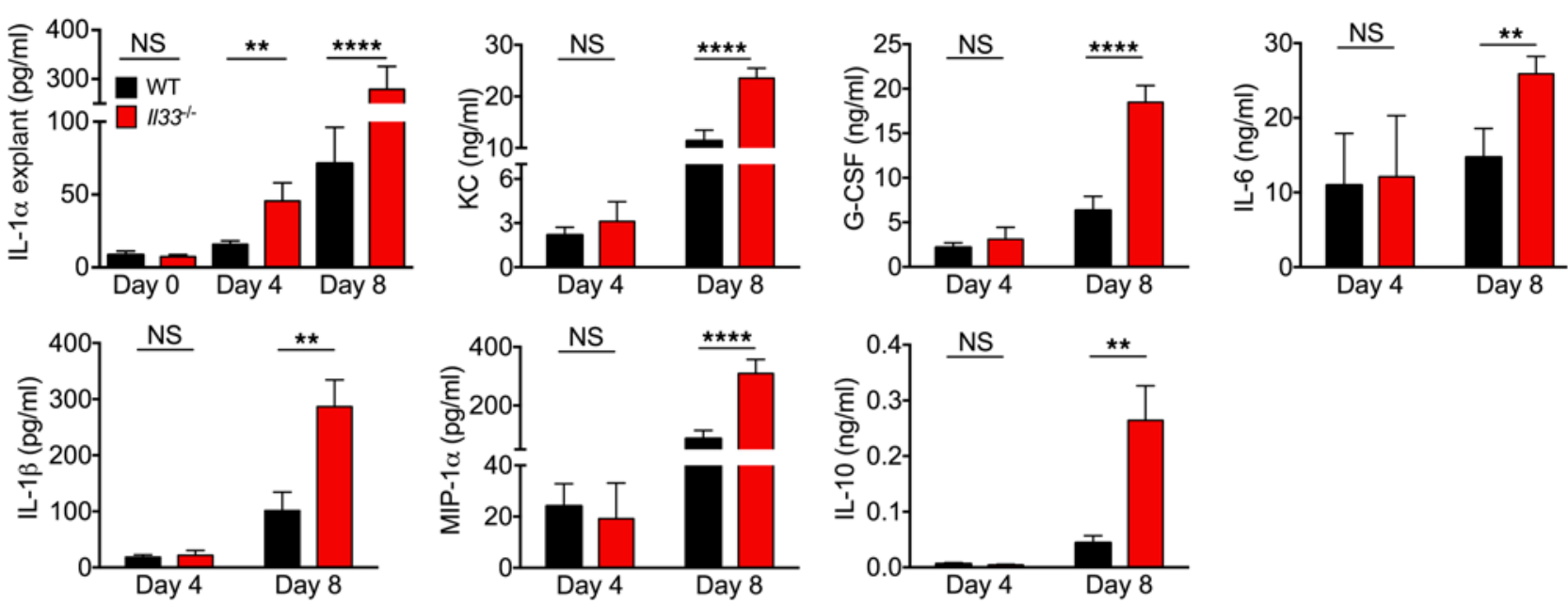

B

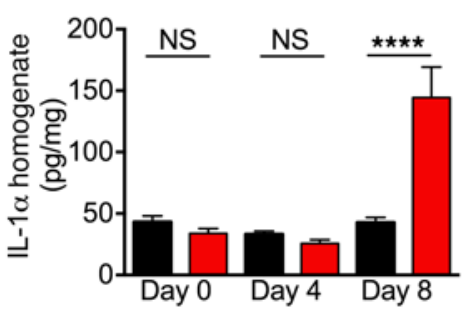

C

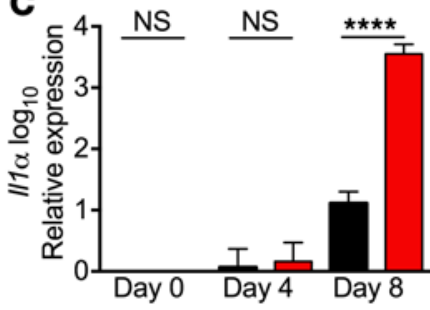

E
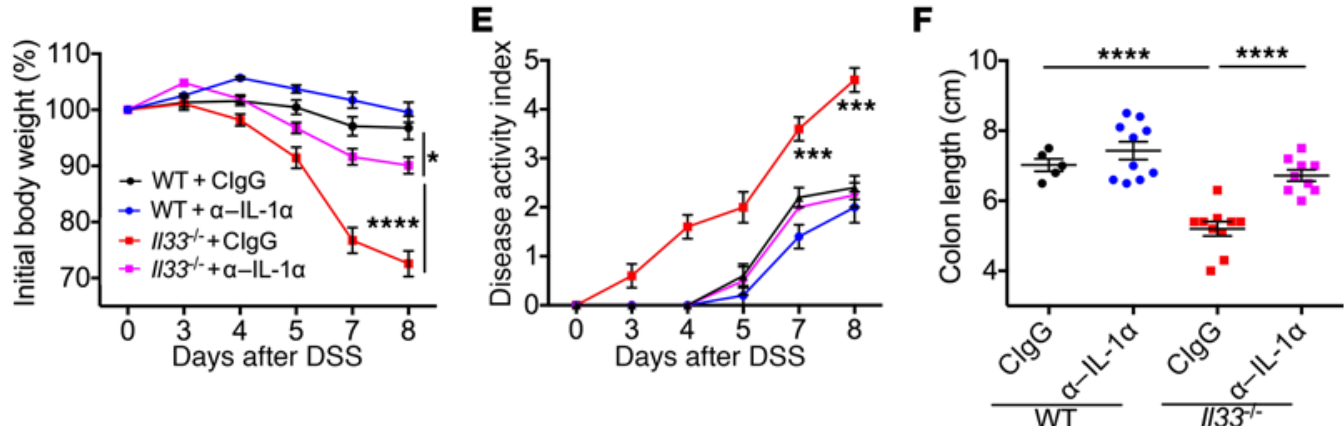

G

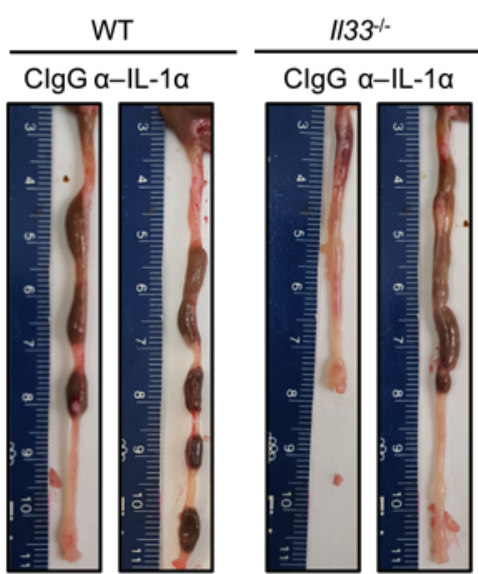

H
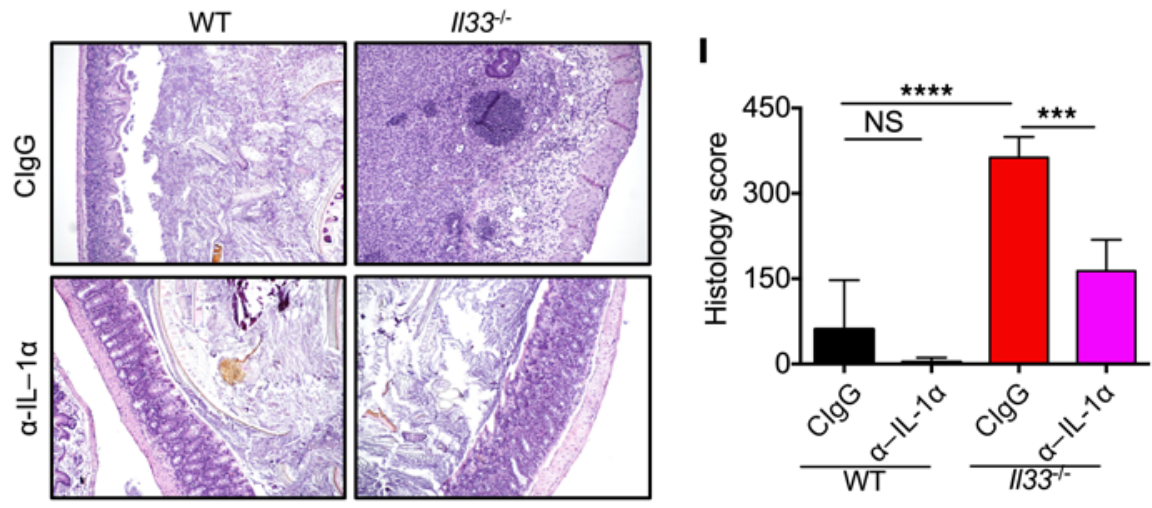

Figure 3. Lack of IL-33 leads to early release of pathogenic IL-1 $\alpha$. See also Supplemental Figure 2. (A) Quantification of cytokines in supernatants of colon explants and (B) quantification of IL-1 $\alpha$ in clarified homogenates of colons of WT and $/ 133^{-/-}$mice at indicated days after DSS administration. (C) qRT-PCR analysis of $1 / 1 a$ expression in whole colon tissue at indicated days after DSS administration. $n=8-10$ mice per time point per group. (D) Body weight loss and (E) disease activity index of WT and $/ 133^{-1-}$ mice during DSS and control IgC or anti-IL-1 $\alpha$ antibody administration. (F) Colon length measurement and (C) representative colon images at day 8 after DSS. (H) Representative images of H\&E-stained colon sections and (I) colon histology analysis at day 8 after DSS. Original magnification, $\times 10 . n=5$ mice for WT+ClgG and 9 to 10 mice for other groups. Data represent 2 independent experiments and were analyzed by Kruskal-Wallis test (A, B, C, F, and I) or 2-way ANOVA ( $\mathbf{D}$ and $\mathbf{E})$ followed by Holm-Šídák post test. Error bars represent mean \pm SEM, and each symbol represents an individual mouse. ${ }^{*} P<0.05 ;{ }^{* *} P<0.01 ;{ }^{* *} P<0.001 ;{ }^{* * *} P<0.0001$. 
A

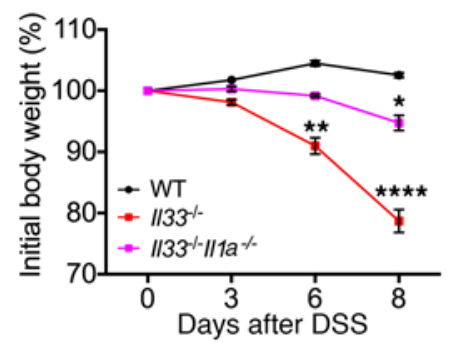

B



C

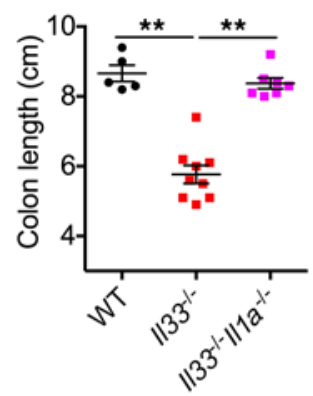

E

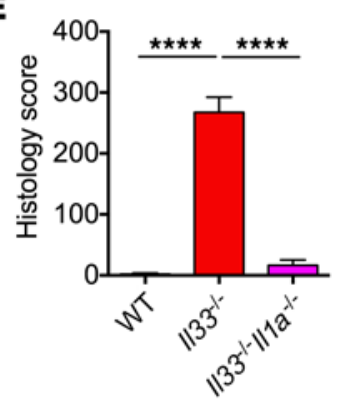

G

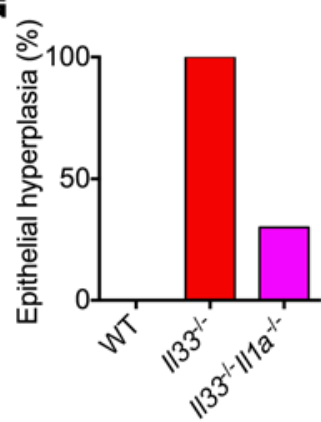

I

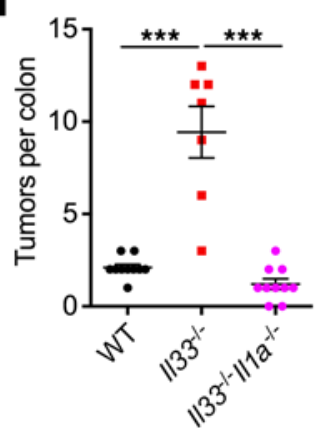

$\mathbf{F}$
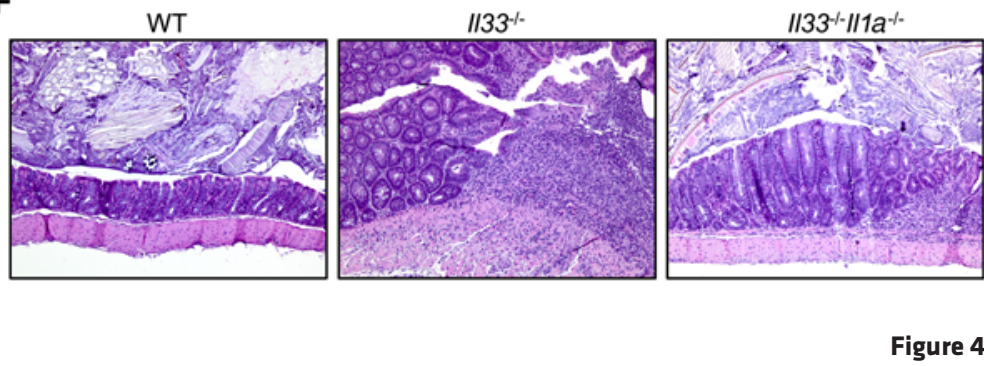

D



H

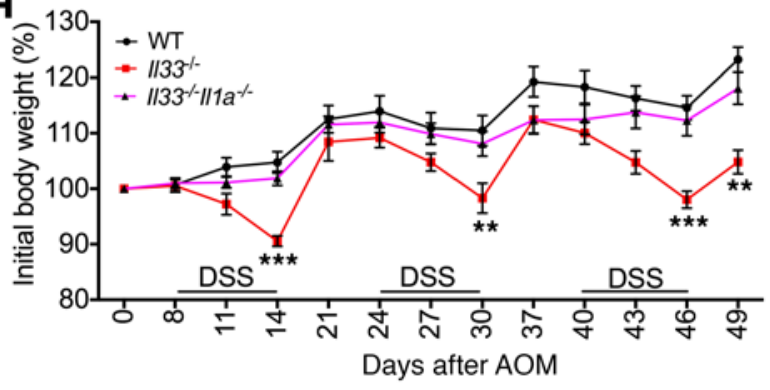

J

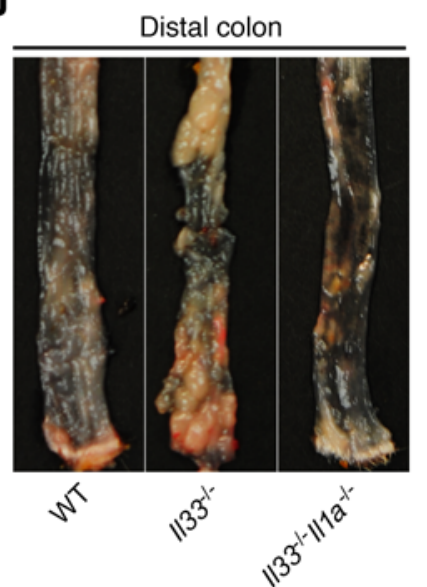

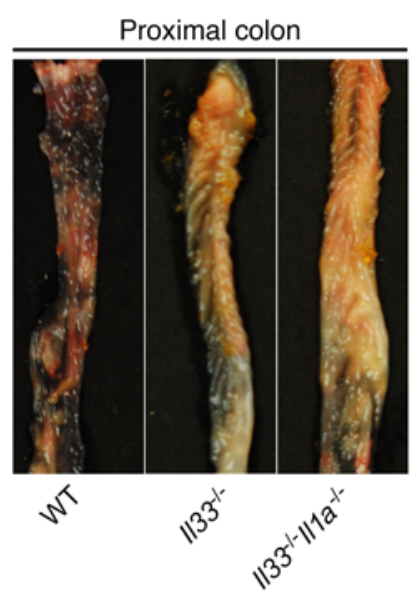

Figure 4. Genetic ablation of Il1a prevents colitis and CAC in $/ 133^{-1-}$ mice. (A) Body weight loss and (B) disease activity

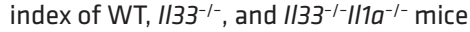
during AOM/DSS administration. (C) Colon length measurement and (D) representative colon images at day 8 after DSS. (E and $\mathbf{G}$ ) Colon histology analysis at day 14 after AOM and (F) representative images of $\mathrm{H} \& \mathrm{E}$-stained colon sections. Original magnification $\times 10$. $n=5$ (WT); $n=9\left(1 / 33^{-/-}\right) ; n=7$ for $\left(1 / 33^{-1-}\right.$ $\left.117 a^{-/-}\right)$. (H) Body weight loss of WT and $1133^{-1-}$ mice injected with AOM on day 0 and administered 3 rounds of 2\% DSS in drinking water. (I) Quantification of the number of tumors in the colon at day 49 after AOM injection (J) Representative images of distal and proximal colon at day 49 after AOM administration. Original magnification, $\times 10 . n=10(\mathrm{WT})$; $n=7\left(I / 33^{-1-}\right) ; n=10\left(I / 33^{-1-1} / 11 a^{-1-}\right)$. Data represent 2 independent experiments and were analyzed by Kruskal-Wallis test (C, E, and I) or 2-way ANOVA (A, B, and H) followed by Holm-Šídák post test. Error bars represent mean \pm SEM, and each symbol represents an individual mouse. ${ }^{*} P<0.05 ;{ }^{*} P<0.01$; ${ }^{* * *} P<0.001 ;{ }^{* * *} P<0.0001$. epithelia and antigen-presenting cells $\left(\mathrm{CD}^{4} 5^{+} \mathrm{CD} 90^{-} \mathrm{MHCII}^{+}\right)$ and $\mathrm{CD} \mathrm{O}^{+}$lymphocytes $\left(\mathrm{CD}^{4} 5^{+} \mathrm{CD} 90^{+} \mathrm{MHCII}\right)$ from the lamina propria. IL-33 was detectable in the colon tissue of WT mice under homeostatic conditions and was increased at both protein and RNA levels at day 8 (Supplemental Figure 2, A and B and Supplemental Methods), consistent with previous reports $(24,25)$. Increased $I l 33$ expression was observed in both epithelial cells and immune cells of the epithelial fraction. Within the lamina propria fraction, expression of $I l 33$ was increased in the antigen-presenting cells, but was not detectable in the $\mathrm{CD} 90^{+}$ lymphocytes (Supplemental Figure 2C). Expression of Illa was upregulated by epithelial cells, antigen-presenting cells, and the $\mathrm{CD} 90^{+}$lymphocytes after DSS administration (Supplemental Figure 2C). Therefore, both epithelial and immune cells of the colon contributed to increased production of IL-33 and IL-1 $\alpha$ during DSS treatment.

To determine the mechanism of early IL- $1 \alpha$ release during DSS treatment, we evaluated inflammasome activation by analyz- 
A

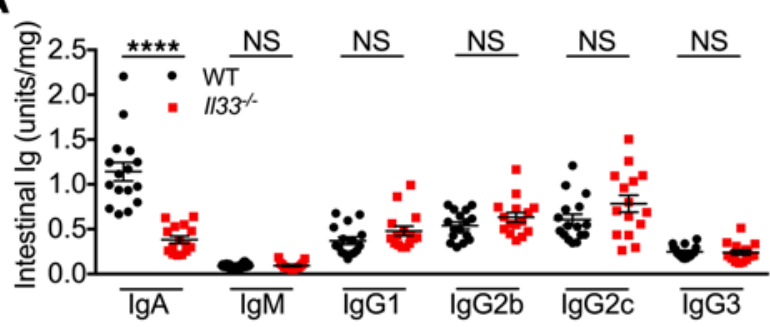

C



D



B

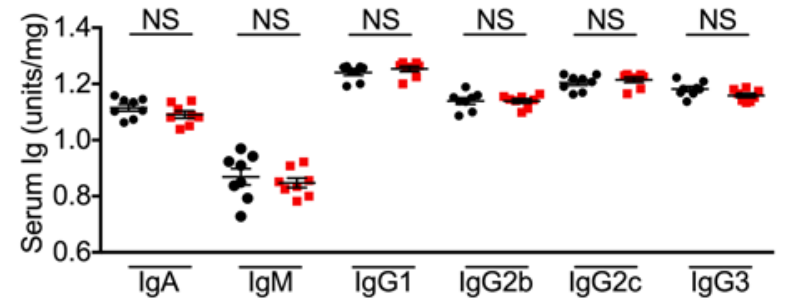

E

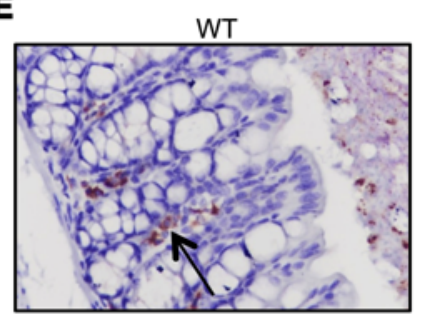

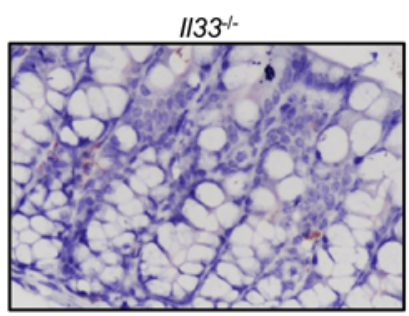

$\mathbf{F}$

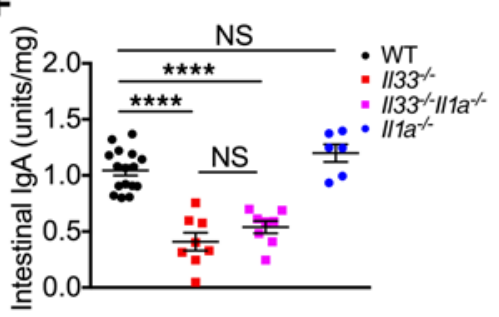

G

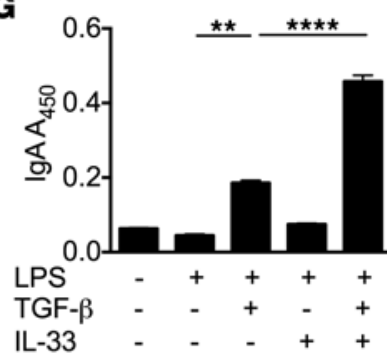

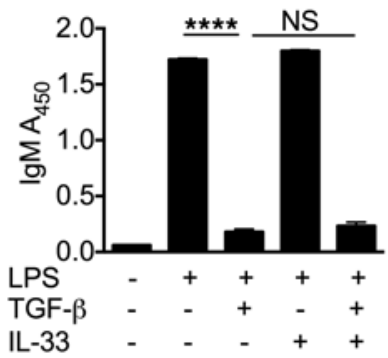

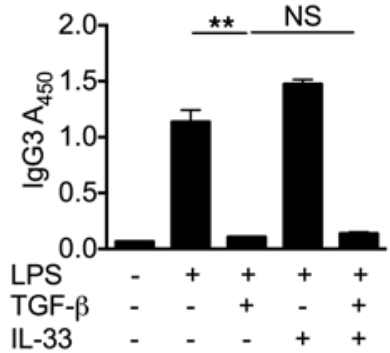

H

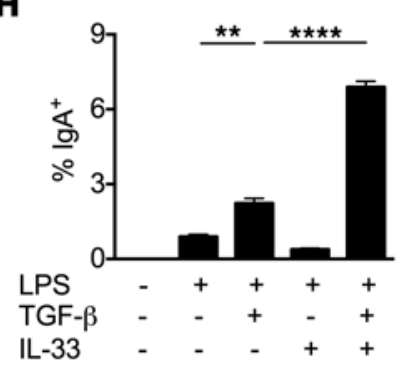

I

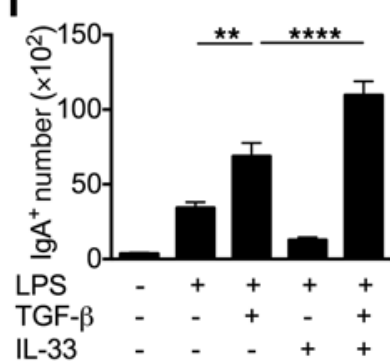

J

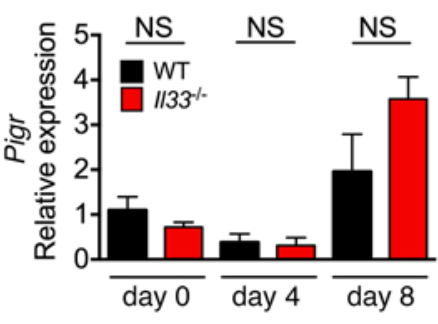

Figure 5. IL-33 promotes intestinal IgA production. (A and B) Immunoglobulin ELISA from colon explants and sera from naive WT and II33-1- mice. (C) Immunoglobulin ELISA from colon explants at days 0, 4, and 8 after AOM administration. (D) Quantification of CD138+1gA+ cells (intracellular stain for IgA) in the colon lamina propria of naive WT and $/ 133^{-/-}$mice by flow cytometry. (E) Representative images for immunohistochemistry for IgA in colon sections

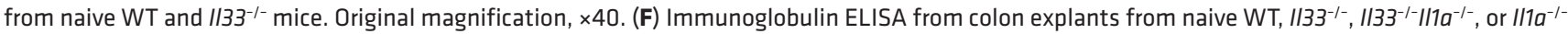
mice. (C) Immunoglobulin ELISA from media supernatants or (H) percentage and (I) number of IgA $A^{+}$B cells from WT splenic B cells (CD19+) treated with LPS, TCF- $\beta$, and/or IL-33 for 4 days. (J) qRT-PCR analysis of Pigr expression in whole colon tissue at indicated days after DSS administration. Data represent 3 independent experiments (A-D, F, and J) or 3 technical replicates that are representative of 3 independent experiments. Data in G-I were analyzed by Kruskal-Wallis test followed by Holm-Šídák post test. Error bars represent mean $\pm \mathrm{SEM}$, and each symbol represents an individual mouse. ${ }^{* *} P<0.01$; ${ }^{* * *} P<0.001 ;{ }^{* * *} P<0.0001$.

ing caspase 1 maturation in the colon homogenates. There was no difference in activation of caspase 1 (observed by the presence of the 10 band) in the colons of WT and $\mathrm{Il33}^{-/-}$mice at day 4 , demonstrating that IL-33 does not regulate inflammasome activation to mediate early IL- $1 \alpha$ release (Supplemental Figure 2D). However, increased ulceration and necrotic cellular morphology were observed at day 4 and day 8 in the colon sections of $I l 33^{-/}$mice after DSS administration (Figure 1, H and I, and Supplemental Figure $2 \mathrm{E}$ ), suggesting that enhanced cell death leads to early IL-1 $\alpha$ release from the $\mathrm{Il} 33^{-/-}$colonocytes.

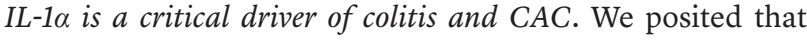
the enhanced IL- $1 \alpha$ release is a critical instigating event in the induction of colitis in $\mathrm{Il33}^{-/-}$mice. To test this hypothesis, IL-1 $\alpha$ was depleted in the $I l 33^{-/-}$mice during early time points of DSS administration by injecting IL- $1 \alpha$-neutralizing antibody. AntiIL-1 $\alpha$ treatment significantly ameliorated body weight loss and other clinical signs of the disease in $\mathrm{Il} 33^{-/-}$mice (Figure 3, D and E). Anti-IL-1 $\alpha$ treatment also significantly protected $I l 33^{-/-}$mice from colon shortening and histological changes associated with DSS administration (Figure 3, F-I). 
Similar to results with antibody-mediated neutralization of IL-1 $\alpha$, mice deficient in both Il33 and Il1a $\left(\mathrm{Il}_{33^{-/}} \mathrm{Illa}^{-/-}\right)$were protected from AOM and DSS-induced body weight loss, clinical signs of disease, colon shortening, and histological changes when compared with $\mathrm{Il33}^{-/-}$mice (Figure 4, A-E). Epithelial hyperplasia is an early event in tumorigenesis, which normally correlates with the extent of inflammation after AOM and DSS treatment. We therefore evaluated the colonic epithelial hyperplasia in WT, $\mathrm{Il33^{-/ }}$, and $\mathrm{Il33^{-/- }} \mathrm{IlIa}^{-/-}$mice at day 14 of AOM and DSS treatment. Consistent with decreased inflammation in the colons of $\mathrm{Il33}^{-1}$ $I l 1 a^{-/-}$mice, a lower proportion of $I l 33^{-/-} I l 1_{1}{ }^{-/-}$mice showed epithelial hyperplasia (30\%) when compared with $I l 33^{-/-}$mice $(100 \%)$ (Figure 4, E-G). Therefore, genetic ablation of Il1a also protects Il33 $3^{-1-}$ mice from epithelial hyperplasia. To specifically determine whether tumor development in $\mathrm{Il33}^{-/-}$mice is decreased by Illa ablation, we treated WT, Il33-/-, and $I l 33^{-1-} I l 1 a^{-/}$mice with AOM followed by 3 rounds of DSS. Il33-/Il1 $a^{-/-}$mice lost significantly lower body weight when compared with $\mathrm{Il33^{-/- }}$ mice during each round of DSS administration (Figure $4 \mathrm{H}$ ). Consistent with decreased hyperplasia at day $14, I l 33^{--1} I l 1 a^{-/-}$mice also harbored a significantly reduced number of tumors in their colons at day 49 when compared with $\mathrm{Il33^{-/- }}$ mice (Figure 4, I and J). The number of tumors in $\mathrm{Il33}^{-/-} \mathrm{Illa}^{-/-}$mice was in fact similar to that in WT mice. Therefore, epithelial hyperplasia data at day 14 and tumor burden at day 49 clearly demonstrate that genetic ablation of Illa protects $\mathrm{I} l 33^{-/-}$mice from CAC.

$\mathrm{Illa}^{-/-}$mice themselves were protected from acute colitis after 4\% DSS administration (Supplemental Figure 3, A and B), consistent with a previous report (28). Moreover, upon AOM and DSS treatment with a $3.5 \%$ DSS dose, $I l 1 a^{-/-}$mice lost significantly less body weight than WT controls at chronic time points of days 66 to 90 (Supplemental Figure 3C). Histological analysis revealed reduced inflammation and epithelial dysplasia in the $\mathrm{Illa}^{-/}$colon (Supplemental Figure 3, D-G). Therefore, IL-1 $\alpha$ is an important driver of colitis and CAC.

IL-33 promotes intestinal IgA production. Next, we examined the mechanism behind increased IL-1 $\alpha$ release from $\mathrm{Il33}^{--}$colonocytes after DSS administration. In order to reach the colonocytes, DSS must first penetrate the mucus barrier. IL-33 has been shown to upregulate the production of mucus after helminth challenge and oxazolone treatment $(14,29)$. We therefore examined possible defects in the mucus production in $\mathrm{I} 33^{-/-}$mice. There were no significant differences in the expression of mucins $M u c 1, M u c 2$, Muc3, Muc4, or Muc5ac at basal or preclinical time points during DSS administration between WT and Il33-/- colons (Supplemental Figure 4A). Further, the number of goblet cells was similar between the colons of WT and $I l 33^{-/-}$mice under homeostatic conditions (day 0). Also, consistent with increased colitis in $\mathrm{Il33}^{-/-}$mice, frequency of goblet cells was decreased in the colons of $\mathrm{Il33}^{-/-}$mice during DSS treatment (Supplemental Figure 4, B and C). IL-33 has also been shown to be an amplifier of type 2 cytokine response from $\mathrm{T}_{\mathrm{H}} 2$ and ILC2 cells after helminth and allergen challenge $(14,29)$. Monticelli et. al. showed that exogenous IL-33 administration expands ILC2s in the GALT and upregulates amphiregulin production (24). However, the impact of IL-33 deficiency on ILC2 and $\mathrm{T}_{\mathrm{H}} 2$ response in the gut under homoeostatic conditions or during DSS administration has not been directly tested. The levels of cytokines associated with ILC2 and $\mathrm{T}_{\mathrm{H}} 2$ response, IL-5, IL-13, and amphiregulin, were similar in the colons of WT and $\mathrm{Il33^{-/ }}$ mice at day 0 and day 4 and increased in the colons of $\mathrm{Il33}^{-/-}$mice at day 8 (Supplemental Figure 5A). We also evaluated the number of ILC2s $\left(\mathrm{CD} 19^{-} \mathrm{CD}^{-} 5^{+} \mathrm{Lin}^{-} \mathrm{CD} 90.2^{+} \mathrm{CD} 127^{+} \mathrm{GATA}^{-3^{+}}\right.$) and $\mathrm{T}_{\mathrm{H}} 2$ cells $\left(\mathrm{CD} 19^{-}\right.$ $\left.\mathrm{CD} 45^{+} \mathrm{Lin}^{+} \mathrm{CD} 90.2^{+} \mathrm{CD} 127^{+} \mathrm{GATA}-3^{+}\right)$in the colons of WT and $\mathrm{Il} 33^{-1-}$ mice during DSS administration. We found that the number of ILC2 and $\mathrm{T}_{\mathrm{H}} 2$ cells in the colons of $I l 33^{-/-}$mice were similar to WT levels at day 0 . At day 8 , the number of ILC2s remained similar, but the number of $\mathrm{T}_{\mathrm{H}} 2$ cells was increased in the colons of $\mathrm{Il33^{-/- }}$ mice (Supplemental Figure 5, B-D). Increase in the number of $\mathrm{T}_{\mathrm{H}} 2$ cells correlated with increased levels of $I l 5$ and Il13 and the extent of inflammation in the $\mathrm{I} 33^{-/-}$mice. These data are consistent with findings from Waddell et. al., who showed that the colonic type 2 cytokine response is preserved in $\mathrm{Il33}^{-/-}$mice after oxazolone treatment (29).

Expression of epithelium-healing genes, such as $I l 22$ and its associated antimicrobial peptides (AMPs) Reg3g and Reg3b, was also unperturbed at day 4 of DSS administration (Supplemental Figure 6A). Furthermore, expression of other AMPs such as lipocalin, pentraxin, and S100a9 (Supplemental Figure 6B) and epithelial tight junction proteins occludin and ZO1 (Supplemental Figure 6C) were also similar between the colons of WT and $\mathrm{Il} 33^{-/-}$mice until day 8 (Supplemental Figure 6, A-C). Schiering et al. have also shown that IL-33 promotes differentiation of Tregs and thereby protects lymphopenic mice from colitis resulting from infusion of naive T cells (30). However, there was no defect in the number of Tregs in the colons of $I l 33^{-/-}$mice at day 0 , and they were increased during DSS administration (Supplemental Figure 6D). Collectively, these data demonstrate that perturbation in production of mucins, type 2 cytokines, AMPs, tight junction proteins, and epithelial restitution factors is not observed in the colons of $\mathrm{Il33^{-/- }}$ mice until overt inflammation is established. Therefore, while perturbation in these pathways may promote disease pathogenesis, they are unlikely to be responsible for the early release of pathogenic IL- $1 \alpha$.

Intestinal IgA is a critical factor in promoting gut homoeostasis. Intestinal IgA has been shown to neutralize toxins and preferentially target IBD-promoting gut bacteria for clearance by $\mathrm{M}$ cells and lamina propria macrophages (31). Further, IgA deficiency predisposes humans to gut infections and IBD and increases severity of DSS-induced colitis in mice (32-34). Therefore, we evaluated the level of intestinal IgA in $\mathrm{Il33}^{-/-}$mice. $\mathrm{Il33^{-/- }}$ mice had significantly decreased levels of IgA in colon explants, while the levels of other immunoglobulins - IgM, IgG2b, IgG2c, IgG3, and IgG1 - were similar to WT levels (Figure 5A). Levels of IgA and other immunoglobulins were similar in sera, suggesting a gut-specific role of IL-33 in regulating IgA production (Figure 5B). Furthermore, levels of intestinal IgA stayed low in the $\mathrm{Il33}^{-/-}$mice during DSS administration (Figure $5 \mathrm{C}$ ). Decrease in intestinal IgA was also confirmed by a decrease in the number of IgA-producing plasma cells (gated as $\mathrm{CD} 138^{+} \operatorname{IgA}^{+}$with intracellular staining for IgA) in the colons of $I l 33^{-/-}$mice (Figure 5, D and E). The intestinal IgA level in $I l 1 a^{-/-}$mice was similar to the WT level, while the level in $I l 33^{-1-} I l 1 a^{-/-}$mice was similar to the level in $I l 33^{-/-}$(Figure 5F). Therefore, protection from colitis and CAC that was conferred by IL-1 $\alpha$ blockade or deficiency was independent of modulation of intestinal IgA production. 
A

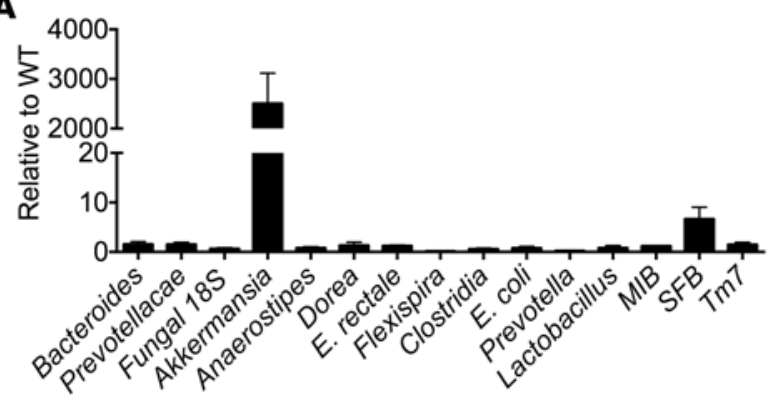

C
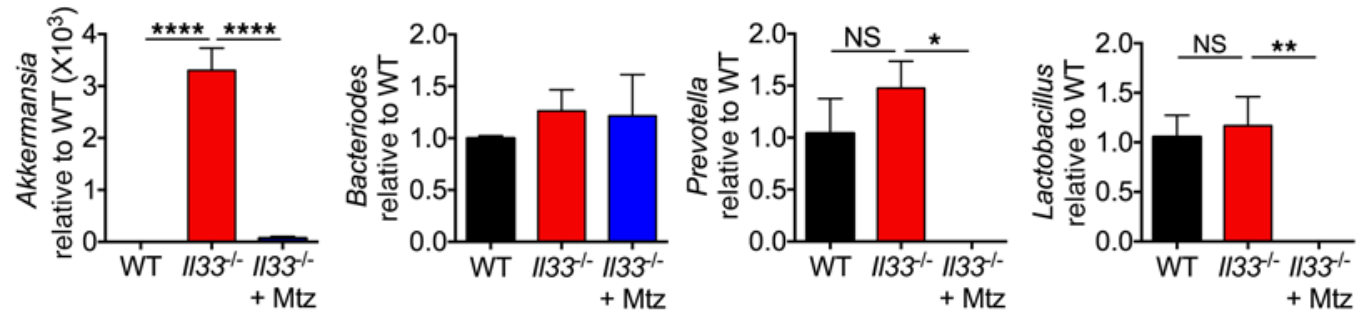
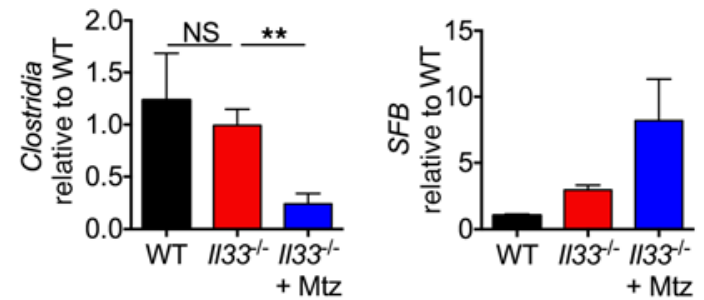

D

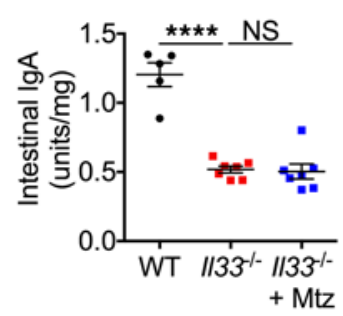

E

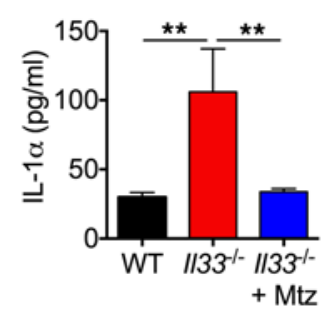

B

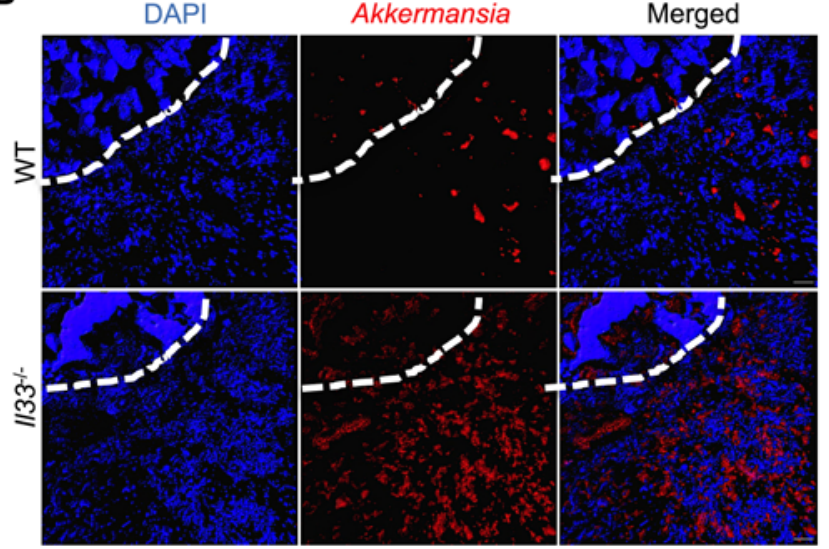

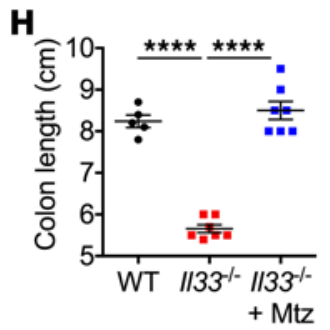

$\mathbf{K}$


\section{J}
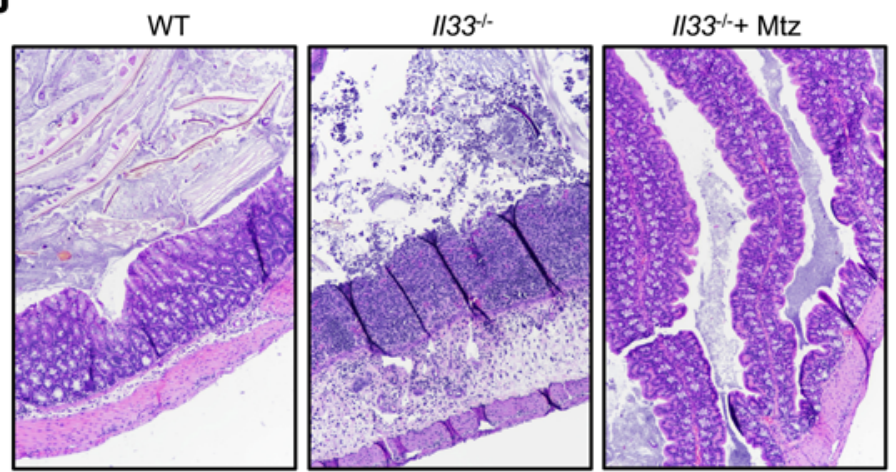
Figure 6. IL-33 regulates gut microbial homeostasis. (A) qPCR analysis of indicated bacteria in the colon contents from naive WT and $/ 133^{-1-}$ mice. (B) Fluorescent in situ hybridization with Akkermansia-specific probe in colon sections from naive mice. Dashed white line represents the epithelium. Scale bars: $8 \mu \mathrm{m}$. (C) qPCR analysis of indicated bacteria from stool samples of naive WT and I/33-1- mice treated with water or metronidazole (Mtz) for 5 days. (D) Quantification of IgA in fecal pellets after 5 days of metronidazole treatment. (E) IL-1 $\alpha$ in supernatants of colon explant cultures at day 4 after DSS administration. (F) Body weight loss and (G) disease activity index of mice during DSS or metronidazole and DSS administration. (H) Colon length measurement and (I) representative colon images at day 8 after DSS or metronidazole and DSS administration. (J) Representative images of H\&E-stained colon sections and (K) colon histology analysis at day 8 after DSS. Original magnification, $\times 10$. Data represent 2 independent experiments and were analyzed by 2 -way ANOVA (F and $\mathbf{G}$ ) or Kruskal-Wallis test (C-E, I, and $\mathbf{K})$, followed by Holm-Šídák post test. Error bars represent mean \pm SEM, and each symbol represents an individual mouse. $n=5(\mathrm{WT}) ; n=7$ for $\left(1 / 33^{-1-}\right)$. ${ }^{*} P<0.05 ;{ }^{* *} P<0.01 ;{ }^{* *} P<0.001 ;{ }^{* * * *} P<0.0001$.

To assess whether IL-33 promotes IgA production from B cells, we utilized an in vitro class-switching assay. WT splenic B cells were stimulated with LPS in the presence of IL-33, TGF- $\beta$, or both. TGF- $\beta$ significantly increased the production of $\operatorname{IgA}$ and decreased production of $\operatorname{IgM}$ and $\operatorname{IgG} 3$ (Figure $5 \mathrm{G}$ ), consistent with previous reports (35). Addition of IL-33 alone did not affect the isotype or quantity of immunoglobulin production. However, addition of both TGF- $\beta$ and IL-33 further enhanced IgA production (Figure $5 \mathrm{G}$ ). Increase in IgA secretion was consistent with increase in both the proportion and total number of $\operatorname{Ig} \mathrm{A}^{+}$cells in $\mathrm{B}$ cells treated with both TGF- $\beta$ and IL-33 (Figure 5, H and I). IL-33, however, did not affect the expression of polymeric immunoglobulin receptor (Pigr), a receptor required for secretion of IgA into the lumen (Figure 5J). Therefore, IL-33 synergizes with TGF- $\beta$ to promote IgA production, and absence of IL-33 leads to decreased IgA levels in the intestine.

Colitis susceptibility in Il33-deficient mice is dependent on dysbiotic microbiota. We and others have shown that gut microbial dysbiosis contributes to susceptibility toward colitis and CAC (36-38). IgA is known to regulate the gut microbial landscape, and mice deficient in IgA and pIgR are known to harbor a dysbiotic microbiota that contributes to their increased susceptibility to colitis $(33,39-41)$. We therefore posited that decreased colonic IgA in $\mathrm{Il}_{33^{-/-}}$mice would lead them to developing a dysbiotic microbiota. To assess whether IL-33 has an effect on gut microbial homeostasis, we performed real-time quantitative PCR (qPCR) analysis of the gut microbiota derived from colon contents of separately housed WT and $\mathrm{Il33}^{-/-}$mice. Relative to WT mice, $\mathrm{Il33}^{-/-}$mice harbored increased levels of Akkermansia muciniphila and segmented filamentous bacteria (SFB), while the levels of bacteria belonging to other class and phyla were similar (Figure 6, A and B). A. muciniphila is an anaerobic bacterium that specializes in degrading the mucus (42) and is highly targeted by intestinal IgA in healthy individuals $(39,41)$. Recent reports have shown that exogenous Akkermansia administration leads to increased permeability of the mucus barrier and epithelial injury in mice after challenge with noxious agents $(43,44)$.

We therefore determined whether increased levels of Akkermansia promote early IL-1 $\alpha$ release and susceptibility of $\mathrm{Il33}^{-/-}$ mice to DSS-induced colitis. Metronidazole is an antibiotic specific to obligate anaerobes (45), and treating $\mathrm{Il33}^{-/-}$mice with metronidazole $(2.5 \mathrm{mg} / \mathrm{ml}$ in $1 \%$ sucrose $)$ for 5 days led to a significant decrease in the level of Akkermansia (Figure 6C). This depletion of Akkermansia by metronidazole treatment was independent of modulation of intestinal IgA level in $\mathrm{Il33^{-/ }}$ mice (Figure $6 \mathrm{D})$, but still led to decreased IL-1 $\alpha$ release from the colon during DSS administration (Figure 6E) and protected the $I l 33^{-/-}$mice from body weight loss, colon shortening, and histological changes associated with DSS administration (Figure 6, F-K). In fact, metronidazole-treated $\mathrm{Il33^{-/- }}$ mice were indistinguishable from WT controls after DSS administration.

Metronidazole treatment also led to a significant decrease in the relative abundance of Lactobacillus and Clostridia, bacterial groups that have been shown to be protective in colitis $(46,47)$, and did not significantly affect the level of SFB (Figure 6C). Therefore, colitis susceptibility in $\mathrm{Il3}^{-/-}$mice is independent of these bacterial groups. Metronidazole administration also decreased the level of Prevotella (Figure 6C), which has been shown to be pathogenic in this model of colitis (48). We therefore employed a strategy to determine the severity of DSS colitis after increasing the level of Akkermansia while simultaneously depleting Prevotella. To this end, mice were treated with a broad-spectrum antibiotic cocktail that we have shown previously to boost the level of Akkermansia, but decrease the level of Prevotella in the gut (49). Pretreatment of mice with this cocktail for 7 days dramatically increased the level of Akkermansia, but decreased Prevotella to a level similar to that seen with metronidazole treatment (Supplemental Figure 7A). Under this microbial landscape, colon explants from WT mice also released increased levels of IL-1 $\alpha$ during DSS administration (Supplemental Figure 7B), and the treated mice displayed increased susceptibility to DSS-induced colitis (Supplemental Figure 7, C-E). Therefore, overrepresentation of the mucolytic bacterium Akkermansia in the $\mathrm{Il33}^{-/-}$mice led to increased IL-1 $\alpha$ release and ensuing colitis after DSS administration.

IL-33 maintains gut homeostasis by modulating the IgAmicrobiota axes. Mice are coprophagus in nature, allowing for equilibration of gut contents by cohousing. Upon cohousing for 2 weeks, levels of Akkermansia equilibrated between cohoused WT and cohoused $\mathrm{Il33}^{-/-}$mice (Figure 7A), which correlated with an increase in levels of IgA in the stool samples of the cohoused Il33-/- mice (Figure 7B). In line with these observations, the cohoused $\mathrm{Il33^{-/ }}$ mice were significantly protected from DSSinduced body weight loss, systemic wasting, and colon shortening (Figure 7, C-E). Histological analysis confirmed amelioration of inflammation and epithelial hyperplasia in the colons of the cohoused $\mathrm{Il33}^{-/-}$mice when compared with the separately housed $\mathrm{Il33}^{-/-}$mice (Figure 7, F and G).

To determine whether IL-33 actively regulates microbial homeostasis, we cohoused WT and $\mathrm{Il} 33^{-/-}$mice for 2 weeks and then separated them for another 4 weeks. While cohousing equilibrated the level of Akkermansia and intestinal IgA in the stool samples, $\mathrm{Il33^{-/- }}$ mice regained the separately housed gut ecological landscape of increased Akkermansia (Figure 7A) and decreased intestinal IgA levels (Figure 7B) after 4 weeks of separation. In 
A



B
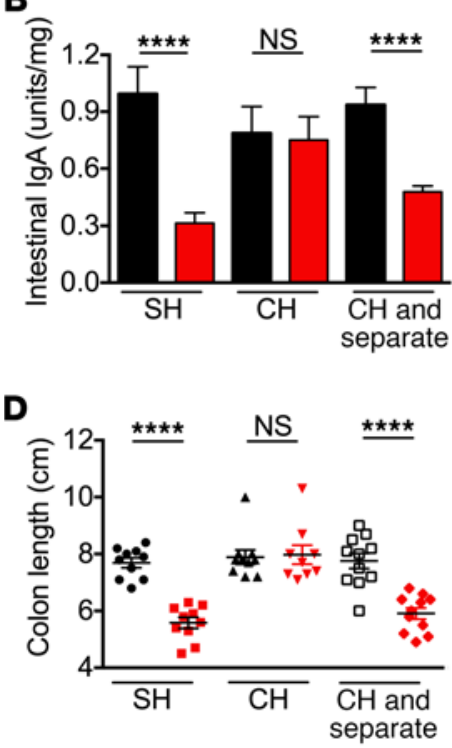

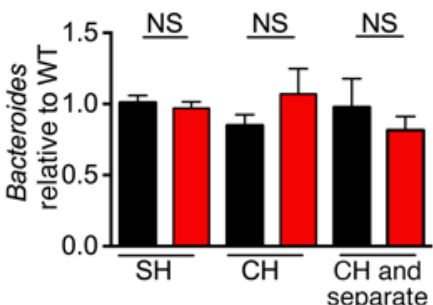

C

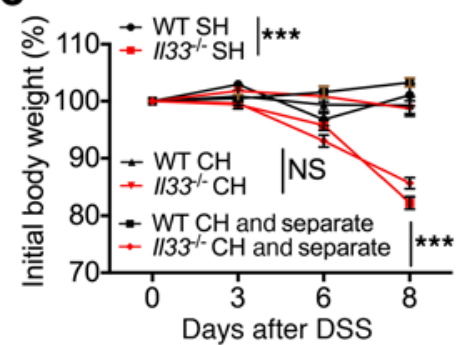

$\mathbf{F}$

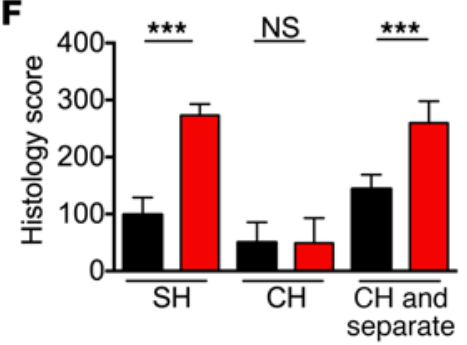

$\mathbf{E}$
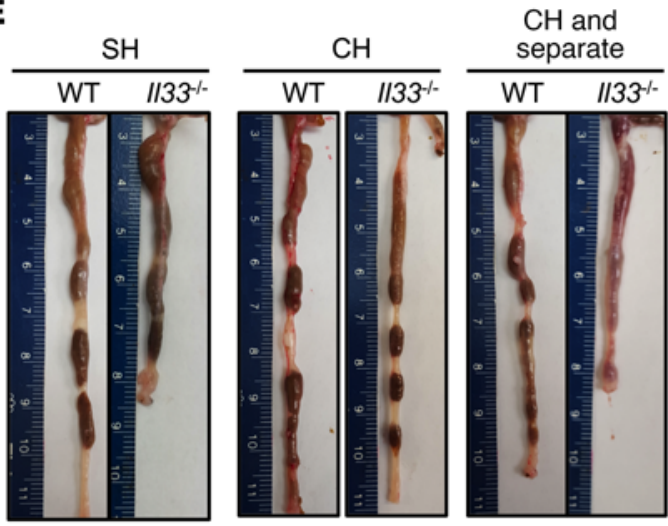

G

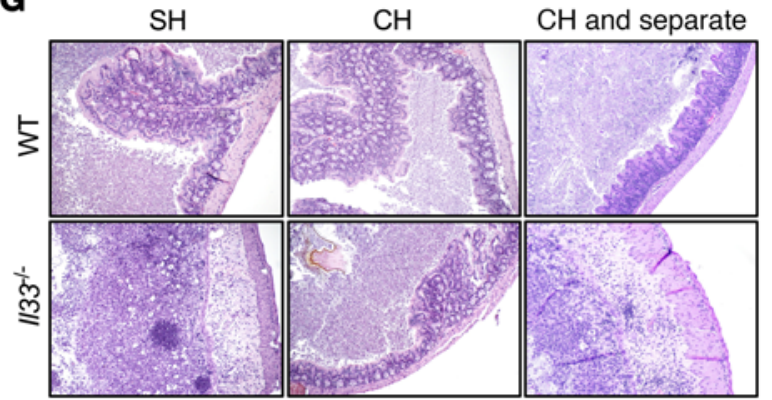

Figure 7. IL-33 actively regulates intestinal IgA levels, microbial homeostasis, and susceptibility to colitis. (A) qPCR analysis of indicated bacteria from stool samples of separately housed (SH), cohoused (CH), or cohoused and then separated (CH and separate) WT and II33-1- mice and (B) IgA measurement in stool samples by ELISA. (C) Body weight change during DSS administration. (D) Colon length measurements and (E) representative colon images at day 8 after DSS administration. (F) Colon histological analysis and (G) representative H\&E-stained colon sections at day 8 after DSS administration. Original magnification, $\times 10$. Data are representative of 2 independent experiments and were analyzed by Kruskal-Wallis test (A, B, D, and F) or 2-way ANOVA (C), followed by Holm-Šídák post test. Error bars represent mean \pm SEM, and each symbol represents an individual mouse. $n=9-11$ mice per group. ${ }^{* *} P<0.001 ;{ }^{* * *} P<0.0001$.

line with these observations, DSS administration led to increased susceptibility toward DSS-induced colitis in the separated $I l 33^{-1-}$ mice (Figure 7, C-G). These data suggest that transfer of intestinal IgA in the $I l 33^{-/}$mice upon cohousing with WT mice actively regulates the load of mucolytic bacteria and confers protection from DSS-induced colitis.

Finally, we used littermate controls to confirm that IL-33 regulates intestinal IgA production and colitis susceptibility independently of the genetic background and starting microbial landscape. $I l 33^{+/-}$mice had WT levels of intestinal IgA and Akkermansia load in the stool, demonstrating that Il33 is haplosufficient for these functions (Supplemental Figure 8 , A and B). In line with these observations, $\mathrm{Il33}^{+/-}$mice were similar to WT controls in susceptibility to DSS-induced colitis (Supplemental Figure 8, C-F). On the other hand, Il33--- littermates that were separated from WT and $\mathrm{Il}_{33^{+/-}}$littermates at weaning for 4 weeks harbored decreased levels of IgA and increased levels of Akkermansia in the stool samples (Supplemental Figure 8, A and B). In line with these observations, Il33/-- littermates displayed increased susceptibility to DSS-induced colitis (Supplemental Figure 8, C-F). Therefore, IL-33 confers protection from colitis by regulating colonic IgA production and inhibiting microbial dysbiosis.

\section{Discussion}

Only $25 \%$ of patients achieve sustained disease remission with the currently available anti-TNF biologics, while another $30 \%$ require total colectomy (50). Therefore, further investigations into the cytokine networks that underlie IBD are required to develop novel therapeutic strategies. Expression of IL-1 $\alpha$, IL-33, ST2, and SST2 are increased in the mucosa of IBD patients, and SNPs in IL33 and ST2 are associated with the development of IBD (18-22). Other studies that utilized distinct models of colitis or exogenous IL-33 infusion have attributed the functions of upregulating the production of mucus, type 2 cytokines and cells, and Tregs to the IL-33/ST2 signaling pathway $(24,29,30)$. Consistent with these studies demonstrating a protective role of IL-33 in the gut, we show here that $I l 33^{-/-}$mice were more susceptible to DSSinduced colitis. However, $I l 33^{-/-}$mice did not have a defect in the expression of mucins, amphiregulin, type 2 cytokine or cellular response, or in the number of Tregs in the colon under basal conditions or early during DSS administration. Instead, Il33-/- colon explants released high levels of IL-1 $\alpha$ early during disease onset. Increased IL-1 $\alpha$ was pathogenic, as its neutralization or genetic deletion markedly protected $\mathrm{Il} 33^{-/-}$mice from colitis. 
IBD is a strong predisposing factor for CAC, and expression of IL33 and ST2 is increased in low-grade tumors, but decreased in high-grade tumors, suggesting a protective role of IL-33 in CAC (22). In line with this hypothesis, we showed that Il33-deficient mice were highly susceptible to AOM/DSS-induced CAC. Furthermore, we showed that IL-1 $\alpha$ was a major driver of colitis and CAC in both Il33-deficient and Il33-sufficient settings. Maywald et al. showed that IL-33 signaling promotes small intestinal tumorigenesis in a model that is dependent on aberrant Wnt signaling instead of inflammation (51). Therefore, the role of IL-33 in restraining colitis-associated colon cancer is due to its dominant role in preventing IL-1 $\alpha$-mediated overt inflammation. IL-1 $\alpha$-blocking monoclonal antibody was well tolerated in phase I clinical trials for psoriasis and solid tumors $(52,53)$. Therefore, further trials in testing its efficacy in ameliorating IBD and CAC are warranted.

Immune system components can regulate the host microbiota that modulate the susceptibility to colitis and CAC $(38,48,54,55)$. Intestinal IgA is one such immune component that preferentially targets colitogenic members of the microbiota, including SFB and A. muciniphila $(34,39,41,56,57)$. Reciprocally, certain pathobionts can produce proteolytic enzymes that degrade intestinal IgA and thereby enhance colitis susceptibility (40). We showed here that IL-33 was essential for efficient production of IgA in the intestine, even under homoeostatic conditions. Similar to the role of IL-33 in Treg differentiation (30), IL-33 promoted IgA production in a TGF- $\beta$-dependent manner. Consistent with decreased levels of intestinal IgA in $\mathrm{Il33}^{-/-}$mice, they also harbored a dysbiotic microbiota associated with increased levels of SFB and, in particular, A. muciniphila. Verrucomicrobia family member Akkermansia expresses mucinases that allow it to degrade the inner mucus layer and utilize it as a carbon and nitrogen source (42). As a consequence, experimental Akkermansia colonization weakens the mucus barrier and promotes epithelial injury after administration of the cytotoxic agent heme (43). Repeated dosing with Akkermansia also leads to increased severity of DSS-induced colitis in WT mice (44) and promotes colon tumorigenesis in a mouse model of aberrant Wnt signaling in the epithelium (58). In line with these studies, we found that depletion of Akkermansia by metronidazole treatment protected Il33-deficient mice from DSS-induced colitis and associated epithelial hyperplasia.

It is of particular clinical relevance that Akkermansia has also been shown to improve obesity and metabolic disorders (59). We have also previously shown that the antibiotic cocktail that increases the levels of Akkermansia also ameliorates IL-1 $\beta$-mediated autoinflammation (49). We showed here that the same antibiotic cocktail also strikingly increased colitis severity. Further, it was recently shown that oral delivery of IgA reactive to pathobionts can decrease colitis susceptibility (39). Therefore, along with modulation of IL-1 $\alpha$ and IL-33 levels, discovering tools that lead to specific manipulation of the microbiota, such as a tailored diet, targeted antibiotic regimen, or oral IgA supplementation, holds great promise of ameliorating inflammatory diseases and cancer.

\section{Methods}

Mice. C57BL/6 WT and $I l 1 a^{-/-}$(60) mice were described previously. Il33 ${ }^{\text {tm1(KомP) Vlcg }}$ (Il33-deficient) embryos were obtained from the
Knock-out Mouse Project (KOMP; https://www.komp.org/) and rederived in $\mathrm{C} 57 \mathrm{BL} / 6$ mice in the colony. Mice were maintained in a specific pathogen-free facility.

Induction of colitis and tumors. For DSS models of colitis, mice were administered the indicated dose of DSS (molecular weight 36-40 kDa; Affymetrix) for 6 days, followed by regular water. For neutralization of IL-1 $\alpha$, mice were injected with $100 \mu \mathrm{g}$ of anti-IL-1 $\alpha$ antibody (clone ALF161 , Bio X Cell) in $100 \mu$ l sterile PBS on days 1,3 , and 5 via retro-orbital route during DSS administration. The AOM/DSS model for colorectal tumorigenesis has been described previously (61). For metronidazole treatment, mice were administered with $2.5 \mathrm{mg} / \mathrm{ml}$ metronidazole (Teva Pharmaceuticals) with $1 \%$ sucrose in drinking water. For cohousing experiments, equal numbers of female WT and female $\mathrm{Il}_{33^{-/-}}$mice were housed in the same cage for 2 weeks before DSS administration and remained cohoused for the duration of the experiment. For separation after cohousing experiments, mice were cohoused for 2 weeks, followed by separation for 4 weeks before DSS administration.

Histology and microscopy analysis. Colon tissues were fixed in 10\% formalin, embedded in paraffin, sectioned, and stained with H\&E. Histological analysis for inflammation, epithelial hyperplasia, and tumorigenesis was performed by a board-certified pathologist (P. Vogel) as described previously (62). FISH for Akkermansia was performed by utilizing Alexa Fluor 647-conjugated probe as described previously (63).

Cytokines and immunoglobulin measurement. Cytokines in colon explants, homogenates, and sera were measured by ELISA. Culture of colon explants (64) and protein extraction from frozen colon tissues (62) was described previously. Briefly, for explant culture, $0.5 \mathrm{~cm}$ of the distal colon was incubated in IMDM media supplemented with $10 \% \mathrm{FBS}$ and penicillin, streptomycin, and gentamycin for 48 hours. Cytokine levels are presented as pg or $\mathrm{ng} / \mathrm{ml}$ of the clarified supernatant media. Explants were also weighed before incubation, and equivalent data are obtained if the cytokine levels are normalized to their wet weight. The IL-18 ELISA was from eBioscience; multiplex ELISAs were used for all other cytokines (Millipore) according to the manufacturers' instructions. Immunoglobulin ELISA was performed by the C57BL/6-HRP clonotyping system (SouthernBiotech) per the manufacturer's instructions.

Real-time qPCR. RNA was isolated using the RNeasy Kit (SigmaAldrich) per the manufacturer's instructions and converted into cDNA as described earlier (64). Gene expression was assessed using $2 \times$ SYBR Green Master Mix according to the manufacturer's instructions (Applied Biosystems). Sequences for qRT-PCR primers are listed in Supplemental Table 1. qPCR data were analyzed by the $2^{-\Delta \Delta C T}$ method, with $\beta$-actin as the housekeeping gene. For gut microbiota characterization, genomic DNA from feces was extracted using the QiAMP DNA Stool Mini Kit (QIAGEN) with bead beating in tissue lyser (Qiagen) and used for real-time PCR amplification of indicated microbiota components by SYBR Green Master Mix (Applied Biosystems) as described earlier (65). Data were analyzed with the $2^{-\Delta \Delta C T}$ method using universal eubacteria primers as control.

$B$ cell class switching assay. The rbc-depleted splenocytes or sortpurified CD19 ${ }^{+}$B cells from naive C57BL/6 WT mice were cultured in media supplemented with anti-CD40 (1 ng/ml), LPS $(1 \mu \mathrm{g} / \mathrm{ml}$, Invivogen), TGF- $\beta$ (1 ng/ml, R\&D Systems), and/or IL-33 (30 ng/ml, Peprotech) in the indicated combinations. After 4 days in culture, supernatant media were analyzed for immunoglobulins by the C57BL/6-HRP Clonotyping System (SouthernBiotech) per the manufacturer's instruc- 
tions. Supernatant concentrations were 1:10,000 for IgM and 1:100 for IgA and IgGs. Only absorbance values more than 3 SD away from the mean of negative control were considered positive. Cells were used for intracellular staining of IgA (clone mA-E61, eBioscience) and analyzed by flow cytometry.

Statistics. Body weight change data were analyzed by 2-way ANOVA followed by the Holm-Šídák post test. Survival data were analyzed by log-rank (Mantel-Cox) test. Statistical significance for other data sets was determined by parametric or nonparametric tests, where appropriate, and are indicated in the figure legends; $P<0.05$ was considered significant.

Study approval. Animal study protocols were approved by the St. Jude Children's Research Hospital Committee on the Use and Care of Animals.

\section{Author contributions}

$\mathrm{AM}$ and TDK conceptualized the project. Methodology was designed by AM, QZ, DS, and RK. Investigation was by AM, QZ,
DS, RK, and PV. AM, DS, QZ, PV, and TDK performed formal analysis. AM wrote the original draft. AM, DS, and TDK reviewed and edited the manuscript. Funding acquisition was by TDK. TDK provided resources. TDK supervised the project.

\section{Acknowledgments}

This work was supported by the NIH (AI101935, AI124346, AR056296 and CA163507 to TDK) and the American Lebanese Syrian Associated Charities (to TDK). We would like to thank Si Ming Man and Prajwal Gurung for helpful discussions and editing of the manuscript. We would like to apologize to our colleagues whose work could not be cited due to space limitations.

Address correspondence to: Thirumala-Devi Kanneganti, Department of Immunology, St. Jude Children's Research Hospital, MS \#351, 262 Danny Thomas Place, Memphis, Tennessee 38105-3678, USA. Phone: 901.595.3634; E-mail: Thirumala-Devi.Kanneganti@ StJude.org.
1. Kappelman MD, et al. The prevalence and geographic distribution of Crohn's disease and ulcerative colitis in the United States. Clin Gastroenterol Hepatol. 2007;5(12):1424-1429.

2. Kappelman MD, Moore KR, Allen JK, Cook SF. Recent trends in the prevalence of Crohn's disease and ulcerative colitis in a commercially insured US population. Dig Dis Sci. 2013;58(2):519-525.

3. Podolsky DK, Isselbacher KJ. Glycoprotein composition of colonic mucosa. Specific alterations in ulcerative colitis. Gastroenterology. 1984;87(5):991-998.

4. Rhodes JM. Unifying hypothesis for inflammatory bowel disease and associated colon cancer: sticking the pieces together with sugar. Lancet. 1996;347(8993):40-44.

5. Bouma G, Strober W. The immunological and genetic basis of inflammatory bowel disease. Nat Rev Immunol. 2003;3(7):521-533.

6. Grivennikov SI, Greten FR, Karin M. Immunity, Inflammation, and Cancer. Cell. 2010;140(6):883-899.

7. Howlader N, et al., eds. SEER Cancer Statistics Review, 1975-2013, National Cancer Institute. NIH Web site http://seer.cancer.gov/ csr/1975_2013/. Updated September 12, 2016. Accessed September 19, 2016.

8. Pichery M, et al. Endogenous IL-33 is highly expressed in mouse epithelial barrier tissues, lymphoid organs, brain, embryos, and inflamed tissues: in situ analysis using a novel Il-33-LacZ gene trap reporter strain. J Immunol. 2012;188(7):3488-3495.

9. Cayrol C, Girard JP. The IL-1-like cytokine IL-33 is inactivated after maturation by caspase-1. Proc Natl Acad Sci U S A. 2009;106(22):9021-9026.

10. Lüthi AU, et al. Suppression of interleukin-33 bioactivity through proteolysis by apoptotic caspases. Immunity. 2009;31(1):84-98.

11. Louten J, et al. Endogenous IL-33 enhances Th2 cytokine production and T-cell responses during allergic airway inflammation. Int Immunol. 2011;23(5):307-315.

12. Barlow JL, et al. Innate IL-13-producing nuocytes arise during allergic lung inflammation and contribute to airways hyperreactivity. J Allergy Clin Immunol. 2012;129(1):191-198.e1.

13. Kurowska-Stolarska M, et al. IL-33 amplifies the polarization of alternatively activated macrophages that contribute to airway inflammation. Jimmunol. 2009;183(10):6469-6477.

14. Humphreys NE, Xu D, Hepworth MR, Liew FY, Grencis RK. IL-33, a potent inducer of adaptive immunity to intestinal nematodes. JImmunol. 2008;180(4):2443-2449.

15. Hung LY, et al. IL-33 drives biphasic IL-13 production for noncanonical Type 2 immunity against hookworms. Proc Natl Acad Sci U S A. 2013;110(1):282-287.

16. Bonilla WV, et al. The alarmin interleukin-33 drives protective antiviral $\mathrm{CD} 8^{+} \mathrm{T}$ cell responses. Science. 2012;335(6071):984-989.

17. Xu D, et al. IL-33 exacerbates antigen-induced arthritis by activating mast cells. Proc Natl Acad Sci U S A. 2008;105(31):10913-10918.

18. Beltrán CJ, et al. Characterization of the novel ST2/IL-33 system in patients with inflammatory bowel disease. Inflamm Bowel Dis. 2010;16(7):1097-1107.

19. Kobori A, et al. Interleukin-33 expression is specifically enhanced in inflamed mucosa of ulcerative colitis. J Gastroenterol. 2010;45(10):999-1007.

20. Seidelin JB, Bjerrum JT, Coskun M, Widjaya B, Vainer B, Nielsen OH. IL-33 is upregulated in colonocytes of ulcerative colitis. Immunol Lett. 2010;128(1):80-85.

21. Latiano A, et al. Associations between genetic polymorphisms in IL-33, IL1R1 and risk for inflammatory bowel disease. PLoS One. 2013;8(4):e62144.

22. O'Donnell C, et al. An antitumorigenic role for the IL-33 receptor, ST2L, in colon cancer. Br J Cancer. 2016;114(1):37-43.

23. Oboki K, et al. IL-33 is a crucial amplifier of innate rather than acquired immunity. Proc Natl Acad Sci U S A. 2010;107(43):18581-18586.

24. Monticelli LA, Osborne LC, Noti M, Tran SV, Zaiss DM, Artis D. IL-33 promotes an innate immune pathway of intestinal tissue protection dependent on amphiregulin-EGFR interactions. Proc Natl Acad Sci U S A. 2015;112(34):10762-10767.

25. Groß P, Doser K, Falk W, Obermeier F, Hofmann C. IL-33 attenuates development and perpetuation of chronic intestinal inflammation. Inflamm Bowel Dis. 2012;18(10):1900-1909.

26. Gaudio E, et al. Dextran sulfate sodium (DSS) colitis in rats: clinical, structural, and ultrastructural aspects. Dig Dis Sci. 1999;44(7):1458-1475.

27. Tanaka T, Kohno H, Suzuki R, Yamada Y, Sugie $\mathrm{S}$, Mori H. A novel inflammation-related mouse colon carcinogenesis model induced by azoxymethane and dextran sodium sulfate. Cancer Sci. 2003;94(11):965-973.

28. Bersudsky M, et al. Non-redundant properties of IL- $1 \alpha$ and IL-1 $\beta$ during acute colon inflammation in mice. Gut. 2014;63(4):598-609.

29. Waddell A, et al. IL-33 signaling protects from murine oxazolone colitis by supporting intestinal epithelial function. Inflamm Bowel Dis. 2015;21(12):2737-2746.

30. Schiering C, et al. The alarmin IL-33 promotes regulatory $\mathrm{T}$-cell function in the intestine. Nature. 2014;513(7519):564-568.

31. Palm NW, et al. Immunoglobulin A coating identifies colitogenic bacteria in inflammatory bowel disease. Cell. 2014;158(5):1000-1010.

32. Brandtzaeg P. Update on mucosal immunoglobulin A in gastrointestinal disease. Curr Opin Gastroenterol. 2010;26(6):554-563.

33. Murthy AK, Dubose CN, Banas JA, Coalson JJ, Arulanandam BP. Contribution of polymeric immunoglobulin receptor to regulation of intestinal inflammation in dextran sulfate sodium-induced colitis. J Gastroenterol Hepatol. 2006;21(9):1372-1380.

34. Mirpuri J, et al. Proteobacteria-specific IgA regulates maturation of the intestinal microbiota. Gut Microbes. 2014;5(1):28-39.

35. Coffman RL, Lebman DA, Shrader B. Transforming growth factor beta specifically enhances IgA production by lipopolysaccharidestimulated murine B lymphocytes. J Exp Med. 1989;170(3):1039-1044.

36. Elinav E, et al. NLRP6 inflammasome regulates 
colonic microbial ecology and risk for colitis. Cell. 2011;145(5):745-757.

37. Normand S, et al. Nod-like receptor pyrin domain-containing protein 6 (NLRP6) controls epithelial self-renewal and colorectal carcinogenesis upon injury. Proc Natl Acad Sci U S A. 2011;108(23):9601-9606.

38. Man SM, et al. Critical role for the DNA sensor AIM2 in stem cell proliferation and cancer. Cell. 2015;162(1):45-58.

39. Planer JD, et al. Development of the gut microbiota and mucosal IgA responses in twins and gnotobiotic mice. Nature. 2016;534(7606):263-266.

40. Moon C, Baldridge MT, Wallace MA, Burnham CA, Virgin HW, Stappenbeck TS. Vertically transmitted faecal IgA levels determine extrachromosomal phenotypic variation. Nature. 2015;521(7550):90-93.

41. Kau AL, et al. Functional characterization of IgA-targeted bacterial taxa from undernourished Malawian children that produce diet-dependent enteropathy. Sci Transl Med. 2015;7(276):276ra24 .

42. Derrien M, Vaughan EE, Plugge CM, de Vos WM. Akkermansia muciniphila gen. nov., sp. nov., a human intestinal mucin-degrading bacterium. Int J Syst Evol Microbiol. 2004;54(pt 5):1469-1476.

43. Ijssennagger N, et al. Gut microbiota facilitates dietary heme-induced epithelial hyperproliferation by opening the mucus barrier in colon. Proc Natl Acad Sci U S A. 2015;112(32):10038-10043.

44. Kang CS, et al. Extracellular vesicles derived from gut microbiota, especially Akkermansia muciniphila, protect the progression of dextran sulfate sodium-induced colitis. PLoS One. 2013;8(10):e76520.

45. Ings RM, McFadzean JA, Ormerod WE. The mode of action of metronidazole in Trichomonas vaginalis and other micro-organisms. Biochem Pharmacol. 1974;23(9):1421-1429.
46. Madsen KL, Doyle JS, Jewell LD, Tavernini MM, Fedorak RN. Lactobacillus species prevents colitis in interleukin 10 gene-deficient mice. Gastroenterology. 1999;116(5):1107-1114.

47. Atarashi K, et al. Induction of colonic regulatory $\mathrm{T}$ cells by indigenous Clostridium species. Science. 2011;331(6015):337-341.

48. Elinav E, et al. NLRP6 inflammasome is a regulator of colonic microbial ecology and risk for colitis. Cell. 2011;145(5):745-757.

49. Lukens JR, et al. Dietary modulation of the microbiome affects autoinflammatory disease. Nature. 2014;516(7530):246-249.

50. Nielsen OH. New strategies for treatment of inflammatory bowel disease. Front Med (Lausanne). 2014;1:3.

51. Maywald RL, et al. IL-33 activates tumor stroma to promote intestinal polyposis. Proc Natl Acad Sci U S A. 2015;112(19):E2487-E2496.

52. Coleman KM, Gudjonsson JE, Stecher M. Openlabel trial of MABp1, a true human monoclonal antibody targeting interleukin $1 \alpha$, for the treatment of psoriasis. JAMA Dermatol. 2015;151(5):555-556.

53. Hong DS, et al. MABp1, a first-in-class true human antibody targeting interleukin-1 $\alpha$ in refractory cancers: an open-label, phase 1 doseescalation and expansion study. Lancet Oncol. 2014;15(6):656-666.

54. Levy M, et al. Microbiota-modulated metabolites shape the intestinal microenvironment by regulating NLRP6 inflammasome signaling. Cell. 2015;163(6):1428-1443.

55. Chen GY, Liu M, Wang F, Bertin J, Núñez G. A functional role for Nlrp6 in intestinal inflammation and tumorigenesis. JImmunol. 2011;186(12):7187-7194.

56. Palm NW, et al. Immunoglobulin A coating identifies colitogenic bacteria in inflammatory bowel disease. Cell. 2014;158(5):1000-1010.

57. Zhang H, Sparks JB, Karyala SV, Settlage R, Luo
XM. Host adaptive immunity alters gut microbiota. ISME J. 2015;9(3):770-781.

58. Dingemanse C, et al. Akkermansia muciniphila and Helicobacter typhlonius modulate intestinal tumor development in mice. Carcinogenesis. 2015;36(11):1388-1396.

59. Schneeberger M, et al. Akkermansia muciniphila inversely correlates with the onset of inflammation, altered adipose tissue metabolism and metabolic disorders during obesity in mice. Sci Rep. 2015;5:16643.

60. Matsuki T, Nakae S, Sudo K, Horai R, Iwakura Y. Abnormal T cell activation caused by the imbalance of the IL-1/IL-1R antagonist system is responsible for the development of experimental autoimmune encephalomyelitis. Int Immunol. 2006;18(2):399-407.

61. Zaki MH, Vogel P, Body-Malapel M, Lamkanfi M, Kanneganti TD. IL-18 production downstream of the Nlrp3 inflammasome confers protection against colorectal tumor formation. JImmunol. 2010;185(8):4912-4920.

62. Zaki MH, et al. The NOD-like receptor NLRP12 attenuates colon inflammation and tumorigenesis. Cancer Cell. 2011;20(5):649-660.

63. Collado MC, Derrien M, Isolauri E, de Vos WM, Salminen S. Intestinal integrity and Akkermansia muciniphila, a mucin-degrading member of the intestinal microbiota present in infants, adults, and the elderly. Appl Environ Microbiol. 2007;73(23):7767-7770.

64. Malik A, Sharma D, St Charles J, Dybas LA, Mansfield LS. Contrasting immune responses mediate Campylobacter jejuni-induced colitis and autoimmunity. Mucosal Immunol. 2014;7(4):802-817.

65. Zhu Q, et al. Cutting edge: STING mediates protection against colorectal tumorigenesis by governing the magnitude of intestinal inflammation. JImmunol. 2014;193(10):4779-4782. 